\title{
NUMERICAL ALGORITHMS OF THE LAWRENCE-DONIACH MODEL FOR LAYERED SUPERCONDUCTORS AND THEIR PARALLEL IMPLEMENTATION*
}

\author{
QIANG DU ${ }^{\dagger}$ AND PAUL GRAY
}

\begin{abstract}
The Lawrence-Doniach model is often used for studying vortex dynamics in superconductors which exhibit a layered structure. In solving these model equations numerically, the added degrees of complexity due to the coupling and nonlinearity of the model often warrant the extensive use of high-performance computers for their solution. Approaches for reducing the complexity of the Lawrence-Doniach model and for simplifying the calculation of the numerical solution are presented in this work. Numerical results and benchmarks are included for models involving the motion of vortices due to an applied current and the pinning of vortices due to nonuniform pinning sites (normal inclusions) placed within the material.
\end{abstract}

Key words. superconductivity, Lawrence-Doniach model, layered superconductors, parallel computing, parallel virtual machine

AMS subject classifications. 65N30, 65Y05, 65C20, 35J65, 81Q05, 81-08

PII. S1064827596311566

1. Introduction. An ongoing thread of research in superconductivity is the characterization of vortex dynamics in type-II superconductors. Type-II superconductors exhibit a mixed state, where the material is neither purely superconducting nor entirely normal (nonsuperconducting). In the mixed state, a magnetic field may penetrate the superconducting sample in the form of a quantized fluxoid. This fluxoid consists of a flux line encircled by a vortex of electron current-hence the term "vortex."

The motion and interaction of the vortices in a type-II superconductor induce a current which counters the effects of the superconductivity. For this reason, there has been an ongoing pursuit to find means for pinning vortices, so that the material is able to maintain its superconducting properties at higher temperatures, currents, or applied fields. Typically, this involves the manufacturing of superconducting materials which are nonhomogeneous or anisotropic in their composition. For example, these pinning sites may be generated within a material by irradiating the material with highenergy ions. Additionally, a visible increase in critical current densities under higher fields and temperatures has recently been observed when nanorods are incorporated into films of $\mathrm{Bi}_{2} \mathrm{Sr}_{2} \mathrm{CaCu}_{2} \mathrm{O}_{8}$ [18].

A typical feature of some high- $T_{c}$ superconductors as well as many superconducting devices is their layered structure - consisting of alternating layers of superconducting and non- (or weakly) superconducting materials. A lucid discussion of layered superconductors may be found in the survey [13]. One of the models for

*Received by the editors November 4, 1996; accepted for publication (in revised form) December 23, 1997; published electronically July 9, 1999. This work was partially supported by NSF grant MS-9500718 and by a DAG grant from HKUST.

http://www.siam.org/journals/sisc/20-6/31156.html

${ }^{\dagger}$ Department of Mathematics, Hong Kong University of Science and Technology, Clear Water Bay, Hong Kong (madu@uxmail.ust.hk), and Department of Mathematics, Iowa State University, Ames, IA 50011.

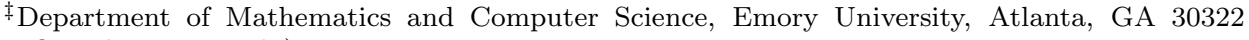
(gray@mathcs.emory.edu). 
layered superconductors is the Lawrence-Doniach (LD) model introduced in [15]; see also $[2,4,9,11,14,16]$ for additional references.

In this paper, numerous issues for the efficient solution of the LD model are discussed. The LD model is a derivative of the basic phenomenological Ginzburg-Landau (GL) theory $[4,17]$ for individual layers and the Josephson-like coupling between the layers. In order to simplify the equations themselves, the LD model is examined in the setting where the superconducting material possesses a high GL parameter $\kappa[6,15]$. The numerical algorithms provided here for the LD model are based on codes developed for the two-dimensional GL equations which have been used extensively in the simulation of vortex dynamics in two dimensions [8]. For the LD model, simplifications are sought which address the complexity introduced by the coupling of the variables between the layers. This interdependence of the variables between layers may be solved for iteratively. The results of this paper show that with a judiciously chosen iterative scheme, the coupling of the variables between the layers may be broken, which allows for a straightforward parallelization of the solution. The convergence of those iterative schemes can be studied rigorously. Numerical tests on various computing platforms such as the DEC-Alpha clusters and the Intel Paragon have shown significant speed-up of the parallel algorithms.

The remaining portion of the paper is outlined as follows. In section 2, notational conventions are given which will be used in subsequent sections. Also given in section 2 are the time-dependent LD equations and their simplifications which are valid for the high- $\kappa$, high-field setting. In section 3 , the details of the discretization of the equations are discussed. Section 4 presents the algorithms posed to decouple and parallelize the model. In section 5, a proof of the convergence of the parallelized algorithms is given. Numerical comparisons of the methods are given in section 6 . In section 7 , simulations of vortex dynamics and vortex torsion are presented along with a discussion, and in section 8 , final comments are given.

2. The time-dependent LD equations. We first briefly explain a few of the terms used to describe the LD model. The GL parameter, $\kappa$, is the ratio of the inplane penetration depth, $\lambda$, and the parallel coherence length, $\xi_{\|}$. These terms yield a measure of distances over which the order parameter and magnetic field, respectively, may undergo appreciable change. ( $\xi_{\perp}$ gives a measure of the change allowed of the order parameter perpendicular to the layers; $\xi_{\|}$measures change allowed within the layer.) For layered superconductors, there is often a strong anisotropy present when one compares material properties parallel and perpendicular to the layers. When the coherence length $\xi_{\perp}$ in the direction perpendicular to the superconducting layer is of the order of the layer spacing, it is necessary to account, in some way, for the discrete nature of the layered structure. In the LD model, the material is treated as a stack of superconducting planes separated by a vacuum or insulating material, and the coupling between the superconducting planes is similar to that which occurs in a Josephson junction. Again, one may consult $[2,4,13,15]$ and the references cited therein for a complete discussion of these models and the physical circumstances necessary for their validity.

The layers are assumed to be perpendicular to the $z$-axis. $\mathcal{D}$ will correspond to a layered material sample such that $\mathcal{D}=\Omega \times[0, S]$, where $\Omega \subset \mathbb{R}^{2}$ is a planar domain and $S$ is the $z$-thickness of the material sample. There are $N+1$ superconducting planes, each having projection $\Omega$ on the $(x, y)$-plane and each separated from its neighbors by a distance $s$; thus, $N s=S$ (see Figure 1). The boundary of $\Omega$ will be 


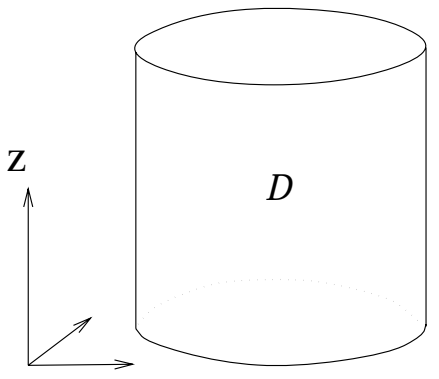

(a)

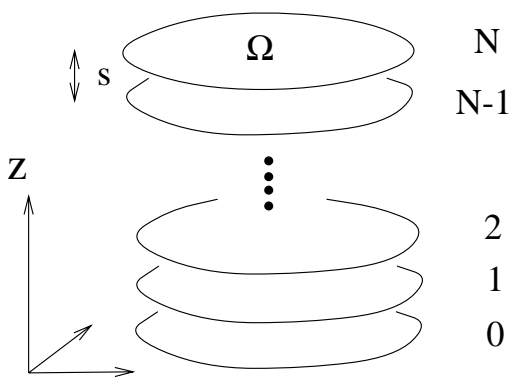

(b)

FIG. 1. A layered material sample depicted in (a) consists of a stack of superconducting layers as depicted in (b).

denoted by $\Gamma$. The region exterior to $\mathcal{D}$ will be denoted by $\mathcal{D}_{e}$, i.e., $\mathcal{D}_{e}=\mathbb{R}^{3} / \overline{\mathcal{D}}$. The interface between $\mathcal{D}$ and $\mathcal{D}_{e}$ will be denoted by $\partial \mathcal{D}$.

Throughout, three-vectors will be denoted by $(\vec{\cdot})$ and two-vectors by boldface notation. Thus, $A, \mathbf{A}$, and $\vec{A}$ denote a scalar, a two-vector, and a three-vector, respectively. There will be occasions where it is convenient to partition a three-vector $\vec{A}$ into the form $\vec{A}=\left(\mathbf{A}, A_{z}\right)^{T}$ so that here $A_{z}$ denotes the third component of $\vec{A}$. The same notational convention will be used for differential operators, for example, $\overrightarrow{\operatorname{grad}}=\left(\operatorname{grad}, \frac{\partial}{\partial z}\right)$.

The LD model uses three primary variables: the order parameter $\psi=\psi(x, y, z)$, whose magnitude is related to the density of superconducting carriers; the magnetic vector potential $\vec{A}=\vec{A}(x, y, z)=\left(\mathbf{A}, A_{z}\right)^{T}$; and $\Phi=\Phi(x, y, z)$, which is the electric potential. We define some auxiliary variables so that

$$
\operatorname{curl} \overrightarrow{A^{a}}=\vec{H}
$$

and

$$
-\sigma \kappa \nabla \Phi^{a}=\operatorname{curl} \vec{H},
$$

where $\sigma$ is a relaxation coefficient used in the model equations. We let $A_{z}$ be the component of $\vec{A}$ in the $z$-direction; $\mathbf{A}_{\mathbf{n}}=\mathbf{A}_{\mathbf{n}}(x, y)=\mathbf{A}(x, y, n s)-\mathbf{A}^{a}(x, y, n s)$, $n=0,1, \ldots, N$, is the restriction of $\mathbf{A}-\mathbf{A}^{a}$ to the $n$th superconducting plane; and similarly $\Phi_{n}=\Phi_{n}(x, y)=\Phi(x, y, n s)-\Phi^{a}(x, y, n s)$ and $\psi_{n}=\psi_{n}(x, y)=\psi(x, y, n s)$. We use similar notation like $\mathbf{A}_{n}^{a}, \Phi_{n}^{a}$ corresponding to the auxiliary variables.

After proper nondimensionalization, the time-dependent LD equations are given by

$$
\begin{gathered}
\frac{\partial \psi_{n}}{\partial t}+i\left(\Phi_{n}+\Phi_{n}^{a}\right) \psi_{n}-\psi_{n}+\left|\psi_{n}\right|^{2} \psi_{n}+\left(\frac{i}{\kappa} \mathbf{g r a d}+\mathbf{A}_{\mathbf{n}}+\mathbf{A}_{\mathbf{n}}^{\mathbf{a}}\right)^{2} \psi_{n} \\
+\rho\left[2 \psi_{n}-\psi_{n+1} \exp \left(-i \phi_{n}^{n+1}\right)-\psi_{n-1} \exp \left(i \phi_{n-1}^{n}\right)\right]=0 \\
\text { in } \Omega \text { and for } n=0,1, \ldots, N,
\end{gathered}
$$

where $\rho$ is a constant coefficient depending on $s$ and $\xi_{\perp}$, and

$$
\phi_{n}^{n+1}=\kappa \int_{n s}^{(n+1) s}\left(A_{z}+A_{z}^{a}\right) d z \quad \text { for } n=0, \ldots, N-1,
$$


and the terms in the bracket describe the Josephson coupling. The characterization of $\psi_{-1}, \psi_{N+1}, \phi_{-1}^{0}$, and $\phi_{N}^{N+1}$ depend on the boundary conditions imposed and/or on the periodicity assumptions of the model. In addition,

$$
\sigma\left(\frac{\partial \vec{A}}{\partial t}+\kappa \nabla \Phi\right)+\operatorname{curl} \overrightarrow{\operatorname{curl}} \vec{A}=\left(\begin{array}{c}
\mathbf{j} \\
j_{z}
\end{array}\right) \quad \text { in } \mathbb{R}^{3},
$$

where $\sigma$ is a material constant representing a driving force. Further, the components of the current are given by

$$
\begin{gathered}
\mathbf{j}=-s \sum_{n=0}^{N}\left(\frac{1}{\kappa} \Im\left\{\psi_{n} \operatorname{grad} \psi_{n}{ }^{*}\right\}+\left|\psi_{n}\right|^{2}\left(\mathbf{A}_{\mathbf{n}}+\mathbf{A}_{\mathbf{n}}^{\mathbf{a}}\right)\right) \delta(z-n s) \text { in } \mathbb{R}^{3}, \\
j_{z}=-2 \kappa \rho s \Im\left\{\psi_{n} \exp \left(i \phi_{n}^{n+1}\right) \psi_{n+1}^{*}\right\}
\end{gathered}
$$

in $\Omega \times[n s,(n+1) s]$ for $n=0, \ldots, N-1(\Im\{\cdot\}$ denotes the imaginary part), and

$$
j_{z}=0 \quad \text { in } \mathcal{D}_{e}=\mathbb{R}^{3} / \overline{\mathcal{D}} .
$$

One also obtains the boundary conditions

$$
\begin{gathered}
\left(\frac{i}{\kappa} \operatorname{grad} \psi_{n}+\mathbf{A}_{\mathbf{n}}^{\mathbf{a}} \psi_{n}\right) \cdot \mathbf{n}=0 \quad \text { on } \Gamma \text { and for } n=0,1, \ldots, N, \\
{[\vec{A} \times \vec{n}]=\mathbf{0} \text { and } \quad[\operatorname{curl} \vec{A} \times \vec{n}]=\mathbf{0} \quad \text { on the boundary } \partial \mathcal{D} \text { of } \Omega \times[0, S],}
\end{gathered}
$$

where [.] denotes the jump at the boundary and

$$
\operatorname{curl}\left(\vec{A}-\vec{A}^{a}\right) \rightarrow \overrightarrow{0} \quad \text { as }|\vec{x}| \rightarrow \infty .
$$

In the high- $\kappa$, high-field setting $[4,11]$, the applied field is assumed to be of the form $\vec{H}=\kappa \vec{H}_{0}$ and $\overrightarrow{A_{0}^{a}}$ is chosen to be a magnetic potential such that curl $\times \vec{A}_{0}^{a}=\vec{H}_{0}$. Then it can be shown that there exists a solution $\psi_{n}, n=0,1, \ldots, N$, and $\vec{A}$ of the LD equations satisfying

$$
\vec{A}=\kappa\left(\vec{A}_{0}+\mathcal{O}\left(\kappa^{-2}\right)\right) \quad \text { and } \quad \psi_{n}=\psi_{0 n}+\mathcal{O}\left(\kappa^{-2}\right), \quad n=0,1, \ldots, N,
$$

where $\psi_{0 n}, n=0,1, \ldots, N$, satisfies

$$
\begin{gathered}
\frac{\partial \psi_{0 n}}{\partial t}+i \Phi_{0 n}^{a} \psi_{0 n}-\psi_{0 n}+\left|\psi_{0 n}\right|^{2} \psi_{0 n}+\left(i \mathbf{g r a d}+\mathbf{A}_{0 n}^{a}\right)^{2} \psi_{0 n} \\
+\rho\left[2 \psi_{0 n}-\psi_{0(n+1)} \exp \left(-i \phi_{0 n}^{n+1}\right)-\psi_{0(n-1)} \exp \left(i \phi_{0(n-1)}^{n}\right)\right]=0 \\
\text { in } \Omega \text { and for } n=0,1, \ldots, N,
\end{gathered}
$$

where

$$
\phi_{0 n}^{n+1}=\int_{n s}^{(n+1) s} A_{0 z}^{a} d z \quad \text { for } n=0, \ldots, N-1,
$$


and

$$
\left(i \operatorname{grad} \psi_{0 n}+\mathbf{A}_{0 n}^{a} \psi_{0 n}\right) \cdot \mathbf{n}=0 \text { on } \Gamma \text { and for } n=0,1, \ldots, N
$$

Equations (2.10)-(2.12) are simplifications of the time-dependent LD model (2.1)(2.9) in the high- $\kappa$, high-field setting. This setting is significant inasmuch as materials made today which maintain their superconducting properties at high temperatures exhibit a large value of $\kappa$. Additional discussions on simplifications in the high- $\kappa$ regime may be found in $[4,6]$. Various numerical algorithms for the solution of (2.10)-(2.12) are discussed in section 3 .

3. The discretization scheme. The numerical scheme is intended to model long-term behavior of the system (2.10)-(2.12). For this reason, the implicit Euler scheme is used for the discretization in time to maintain stability. Letting $\delta_{\Delta t}^{-}$denote the backward difference operator, the discretization in time of the leading order equations leads to the following equation:

$$
\begin{gathered}
\delta_{\Delta t}^{-}\left(\psi_{0 n}\right)+i \Phi_{0 n}^{a} \psi_{0 n}-\psi_{0 n}+\left|\psi_{0 n}\right|^{2} \psi_{0 n}+\left(i \mathbf{g r a d}+\mathbf{A}_{0 n}^{a}\right)^{2} \psi_{0 n} \\
+\rho\left[2 \psi_{0 n}-\psi_{0(n+1)} \exp \left(-i \phi_{0 n}^{n+1}\right)-\psi_{0(n-1)} \exp \left(i \phi_{0(n-1)}^{n}\right)\right]=0 \\
\text { in } \Omega \text { and for } n=0,1, \ldots, N
\end{gathered}
$$

Furthermore, the spatial discretization is done by Galerkin finite element methods, namely, we take the weak form of (3.1) and use finite element spaces as the test and trial spaces $[6,7,8]$. Let $S^{h}$ be a finite element subspace corresponding to a mesh, with mesh parameter $h$. Let $\left\{\psi_{n}^{h}, n=-1, \ldots, N+1\right\}$ be the finite element solution in $S^{h}$. Then, we have

$$
\begin{gathered}
\delta_{\Delta t}^{-}\left(\psi_{n}^{h}, v^{h}\right)+\left(i \Phi_{0 n}^{a} \psi_{n}^{h}, v^{h}\right) \\
+\left(-\psi_{n}^{h}+\left|\psi_{n}^{h}\right|^{2} \psi_{n}^{h}, v^{h}\right)+\left(\left(i \mathbf{g r a d}+\mathbf{A}_{0 n}^{a}\right) \psi_{n}^{h},\left(i \mathbf{g r a d}+\mathbf{A}_{0 n}^{a}\right) v^{h}\right) \\
+\rho\left(\left[2 \psi_{n}^{h}-\psi_{(n+1)}^{h} \exp \left(-i \phi_{0 n}^{n+1}\right)-\psi_{(n-1)}^{h} \exp \left(i \phi_{0(n-1)}^{n}\right)\right], v^{h}\right)=0 \\
\forall v^{h} \in S^{h} \text { and for } n=0,1, \ldots, N,
\end{gathered}
$$

with appropriate conditions on $\psi_{-1}^{h}$ and $\psi_{N+1}^{h}$.

Equation (3.2) may be rewritten as

$$
\begin{aligned}
& \frac{1}{\Delta t}\left(\psi_{n}^{h}, v^{h}\right)+\left(i \Phi_{0 n}^{a} \psi_{n}^{h}, v^{h}\right) \\
& +\left(\left(2 \rho-1+\left|\psi_{n}^{h}\right|^{2}\right) \psi_{n}^{h}, v^{h}\right)\left(\left(i \operatorname{grad}+\mathbf{A}_{0 n}^{a}\right) \psi_{n}^{h},\left(i \mathbf{g r a d}+\mathbf{A}_{0 n}^{a}\right) v^{h}\right) \\
& -\rho\left(\psi_{(n+1)}^{h} \exp \left(-i \phi_{0 n}^{n+1}\right), v^{h}\right)-\rho\left(\psi_{(n-1)}^{h} \exp \left(i \phi_{0(n-1)}^{n}\right), v^{h}\right) \\
& \quad=\frac{1}{\Delta t}\left(\psi_{n, o l d}^{h}, v^{h}\right) \quad \forall \quad v^{h} \in S^{h} \text { and for } n=0,1, \ldots, N,
\end{aligned}
$$

where $\psi_{n, \text { old }}^{h}$ is the solution at the previous time step. In the case with no proximity effect or periodicity assumption, we consider the boundary conditions: $\psi_{N+1}^{h}=$ $\psi_{N}^{h} \exp \left(i \phi_{0 N}^{N+1}\right)$ and $\psi_{-1}^{h}=\psi_{0}^{h} \exp \left(-i \phi_{0(-1)}^{0}\right)$. In this form, the above nonlinear system of equations is easily cast into matrix terms as

$$
\mathbf{M}\left(\mathbf{P}_{n}\right) \mathbf{P}_{n}+\mathbf{C}_{n} \mathbf{P}_{n+1}+\mathbf{C}_{n-1}^{*} \mathbf{P}_{n-1}=\mathbf{F}_{n} \quad \text { for } n=0,1, \ldots, N
$$



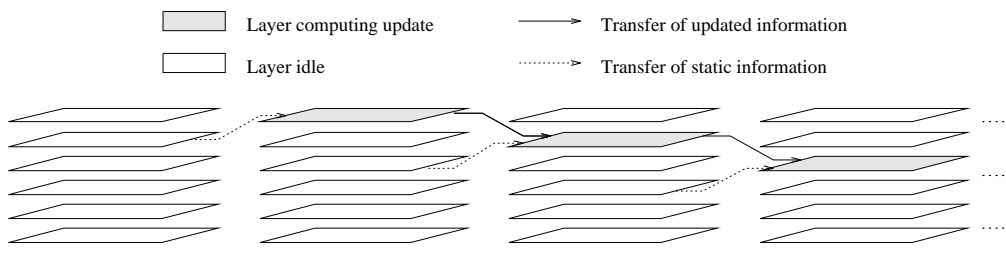

Sequential Update Model
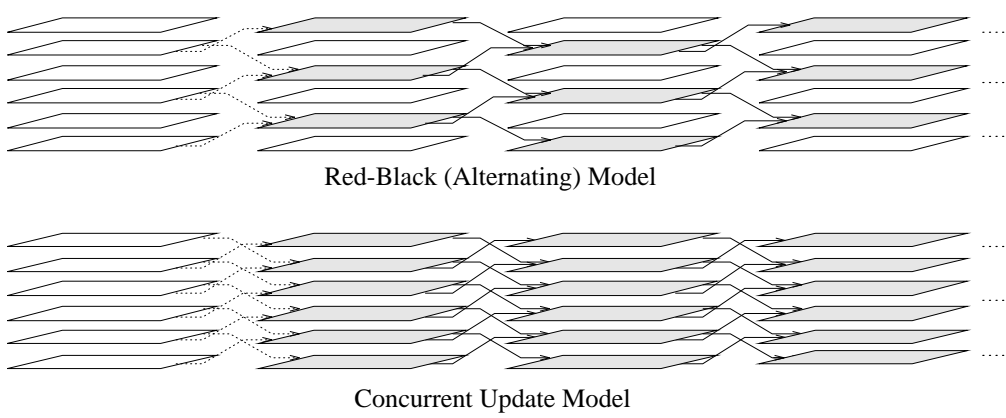

FIG. 2. A pictorial description of iterative schemes used to solve the system (3.3) or, equivalently, (3.4).

where the vector $\mathbf{P}_{n}$ represents the $n$th layer unknowns, $\mathbf{M}\left(\mathbf{P}_{n}\right)$ and $\mathbf{C}_{n}$ represent the coefficient matrices, $\mathbf{C}_{n}^{*}$ is the adjoint matrix of $\mathbf{C}_{n}$, and $\mathbf{F}_{n}$ is associated with the computations involving the previous time step. Denoting $\mathbf{M}_{n}=\mathbf{M}\left(\mathbf{P}_{n}\right)$, one can group all of these variables together to form the nonlinear system

$$
\left(\begin{array}{ccccc}
\mathbf{M}_{0} & \mathbf{C}_{0} & & & \\
\mathbf{C}_{0}^{*} & \mathbf{M}_{1} & \mathbf{C}_{1} & & \\
& \ddots & \ddots & \ddots & \\
& & \mathbf{C}_{N-2}^{*} & \mathbf{M}_{N-1} & \mathbf{C}_{N-1} \\
& & & \mathbf{C}_{N-1}^{*} & \mathbf{M}_{N}
\end{array}\right) \mathbf{P}=\mathbf{F} .
$$

Other types of boundary conditions such as periodicity in the $z$-direction and the De Gennes boundary condition for proximity effect $[3,17]$ may yield slight modifications of the system (3.4).

4. Parallelization of the computational solution. The algorithms for parallelization presented here focus on decoupling the dependence of $\psi_{n}^{h}$ on $\psi_{(n+1)}^{h}$ (from the layer above) and $\psi_{(n-1)}^{h}$ (from the layer beneath) by some modifications of the bracketed portion of (3.2) and thus the corresponding off-diagonal blocks in (3.4).

There are several ways to solve system (3.3) or (3.4). The focus of this paper is the iterative solution of $\psi_{n}^{h}$ or, equivalently, the iterative solution of the vector $\mathbf{P}$ in (3.4). Taking a six-layer model as an example, the physical interpretation of iterative schemes for the solution of (3.4) is given in Figure 2 and their mathematical interpretations are given in the subsequent sections.

4.1. The sequential update model. This model sequentially updates a single layer at one time. Referring to (3.3), $\psi_{n}{ }^{j}$ will be used to denote the $j$ th iterative solution of $\psi_{n}$. In the sequential model presented below, $\psi_{n}{ }^{j}$ is computed using the updated value of the layer beneath it, $\psi_{0(n-1)}^{j}$, and the previous value of the variables 
in the layer above it, $\psi_{0(n+1)}^{j-1}$. Initialization of the method is satisfied by solving the expression

$$
\begin{aligned}
& \delta_{\Delta t}^{-}\left(\psi_{01}^{1}\right)+i \Phi_{01}^{a} \psi_{01}^{1}-\psi_{01}^{1}+\left|\psi_{01}^{1}\right|^{2} \psi_{01}^{1} \\
& +\left(i \text { grad }+\mathbf{A}_{01}^{a}\right)^{2} \psi_{01}^{1}+\rho\left[\psi_{01}^{1}-\psi_{02}^{0} \exp \left(-i \phi_{01}^{2}\right)\right]=0 \quad \text { in } \Omega .
\end{aligned}
$$

This yields $\psi_{01}^{1}(t)$, which is then available for the computation of $\psi_{02}^{1}$. Note that

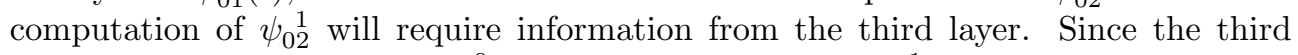
layer has yet to be updated, $\psi_{03}^{0}$ is used for the update of $\psi_{02}^{1}$. In general, the method reverts to solving the equation

$$
\begin{aligned}
& \delta_{\Delta t}^{-}\left(\psi_{0 n}^{j}\right)+i \Phi_{0 n}^{a} \psi_{0 n}{ }^{j}-\psi_{0 n}{ }^{j}+\left|\psi_{0 n}{ }^{j}\right|^{2} \psi_{0 n}{ }^{j}+\left(i \mathbf{g r a d}+\mathbf{A}_{0 n}^{a}\right)^{2} \psi_{0 n}{ }^{j} \\
& \quad+\rho\left[2 \psi_{0 n}^{j}-\psi_{0(n+1)}^{j-1} \exp \left(-i \phi_{0 n}^{n+1}\right)-\psi_{0(n-1)}^{j} \exp \left(-i \phi_{0(n-1)}^{n}\right)\right]=0 \quad \text { in } \Omega .
\end{aligned}
$$

Since each layer must wait until the layer beneath it has completed its update, this model is best suited for a single-processor setting. Note that initialization using the lowest layer is somewhat arbitrary. The method is easily generalized to an arbitrary layer's initialization and a more general sequence of subsequent layer updates. For example, the pictorial representation of the sequential method in Figure 2 shows a top-layer-down update approach.

In matrix terms, this algorithm is similar to the block Gauss-Seidel iteration for

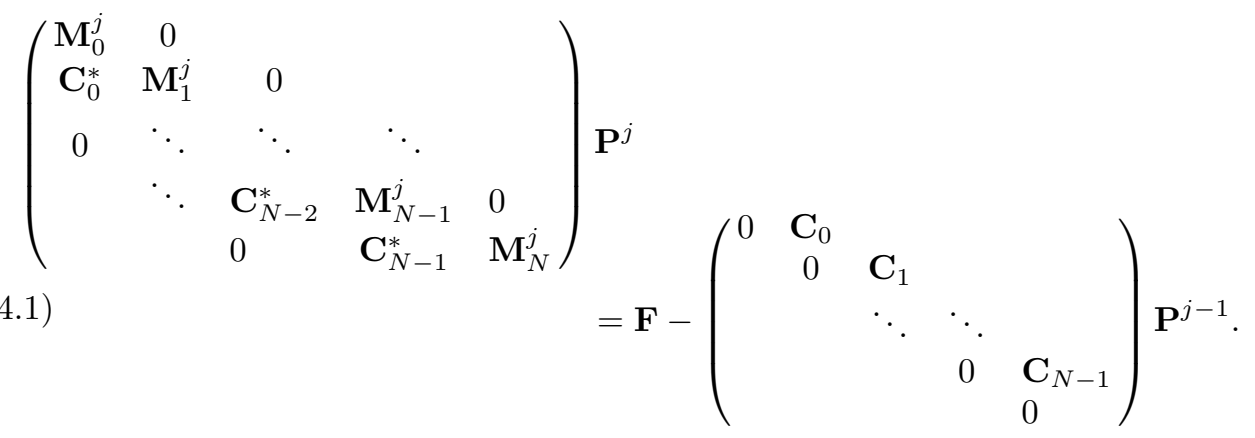

4.2. The red-black model. This model alternately computes updates to every other layer. The model presented below can be loosely described as a computing of the $j$ th iteration of the red layers (the odd layers, for example) with information from the $(j-1)$ th iteration of the black (even) layers. Next, the $j$ th update of the black layers are updated using the updated $j$ th iteration values of the red layers. The method is given as follows:

$$
\begin{aligned}
\delta_{\Delta t}^{-}\left(\psi_{0 n}{ }^{j}\right) & +i \Phi_{0 n}^{a} \psi_{0 n}{ }^{j}-\psi_{0 n}{ }^{j}+\left|\psi_{0 n}{ }^{j}\right|^{2} \psi_{0 n}{ }^{j}+\left(i \operatorname{grad}+\mathbf{A}_{0 n}^{a}\right)^{2} \psi_{0 n}{ }^{j} \\
& +\rho\left[2 \psi_{0 n}^{j}-\psi_{0(n+1)}^{j-1} \exp \left(-i \phi_{0 n}^{n+1}\right)-\psi_{0(n-1)}^{j-1} \exp \left(-i \phi_{0(n-1)}^{n}\right)\right]=0 \\
& \text { in } \Omega \text { if } n \text { red. } \\
\delta_{\Delta t}^{-}\left(\psi_{0 n}{ }^{j}\right)+ & +i \Phi_{0 n}^{a} \psi_{0 n}{ }^{j}-\psi_{0 n}{ }^{j}+\left|\psi_{0 n}{ }^{j}\right|^{2} \psi_{0 n}{ }^{j}+\left(i \mathbf{g r a d}+\mathbf{A}_{0 n}^{a}\right)^{2} \psi_{0 n}{ }^{j} \\
& +\rho\left[2 \psi_{0 n}{ }^{j}-\psi_{0(n+1)}^{j} \exp \left(-i \phi_{0 n}^{n+1}\right)-\psi_{0(n-1)}^{j} \exp \left(-i \phi_{0(n-1)}^{n}\right)\right]=0 \\
& \text { in } \Omega \quad \text { if } n \text { black. }
\end{aligned}
$$


In this model, only the black layers must wait for updated information from the red layers and vice versa. Further, the red-layer and black-layer updates, respectively, may be computed independently. For this reason, the red-black alternating model is well suited for the setting where the material being modeled is characterized by an even number of layers and there is one processor available per two layers of the material. Each processor would be assigned computation of a red layer's update and the subsequent computation of a black layer's update.

Again, in matrix terms, this translates to the two-stage linear system

$$
\begin{aligned}
& \left(\begin{array}{cccc}
\mathbf{M}_{0}^{j} & 0 & & \\
0 & \mathbf{M}_{2}^{j} & 0 & \\
& 0 & \mathbf{M}_{4}^{j} & \ddots \\
& & \ddots & \ddots
\end{array}\right) \mathbf{P}_{\text {red }}^{j}=\mathbf{F}_{\text {red }}-\left(\begin{array}{cccc}
\mathbf{C}_{0} & & & \\
\mathbf{C}_{1}^{*} & \mathbf{C}_{2} & & \\
& \mathbf{C}_{3}^{*} & \mathbf{C}_{4} & \\
& & \ddots & \ddots
\end{array}\right) \mathbf{P}_{\text {black }}^{j-1} \\
& \left(\begin{array}{cccc}
\mathbf{M}_{1}^{j} & 0 & & \\
0 & \mathbf{M}_{3}^{j} & 0 & \\
& 0 & \mathbf{M}_{5}^{j} & \ddots \\
& & \ddots & \ddots
\end{array}\right) \mathbf{P}_{\text {black }}^{j}=\mathbf{F}_{\text {black }}-\left(\begin{array}{cccc}
\mathbf{C}_{1} & & & \\
\mathbf{C}_{2}^{*} & \mathbf{C}_{3} & & \\
& \mathbf{C}_{4}^{*} & \mathbf{C}_{5} & \\
& & \ddots & \ddots
\end{array}\right) \mathbf{P}_{\text {red }}^{j} .
\end{aligned}
$$

4.3. Concurrent update model. In this model, the $j$ th update of each layer is computed using the $(j-1)$ th value of its neighboring layers. The computational algorithm is as follows:

$$
\begin{aligned}
& \delta_{\Delta t}^{-}\left(\psi_{0 n}^{j}\right)+i \Phi_{0 n}^{a} \psi_{0 n}^{j}-\psi_{0 n}{ }^{j}+\left|\psi_{0 n}{ }^{j}\right|^{2} \psi_{0 n}{ }^{j}+\left(i \mathbf{g r a d}+\mathbf{A}_{0 n}^{a}\right)^{2} \psi_{0 n}{ }^{j} \\
& \quad+\rho\left[2 \psi_{0 n}^{j}-\psi_{0(n+1)}^{j-1} \exp \left(-i \phi_{0 n}^{n+1}\right)-\psi_{0(n-1)}^{j-1} \exp \left(-i \phi_{0(n-1)}^{n}\right)\right]=0 \text { in } \Omega .
\end{aligned}
$$

Since each layer's update depends only upon the previous updates of its neighboring layers, each layer update may be computed independently. This makes the simultaneous model best suited to the setting where there is one processor available per layer of the model.

In matrix terms, (4.3) is similar to a block Gauss-Jacobi approach to solving (3.4):

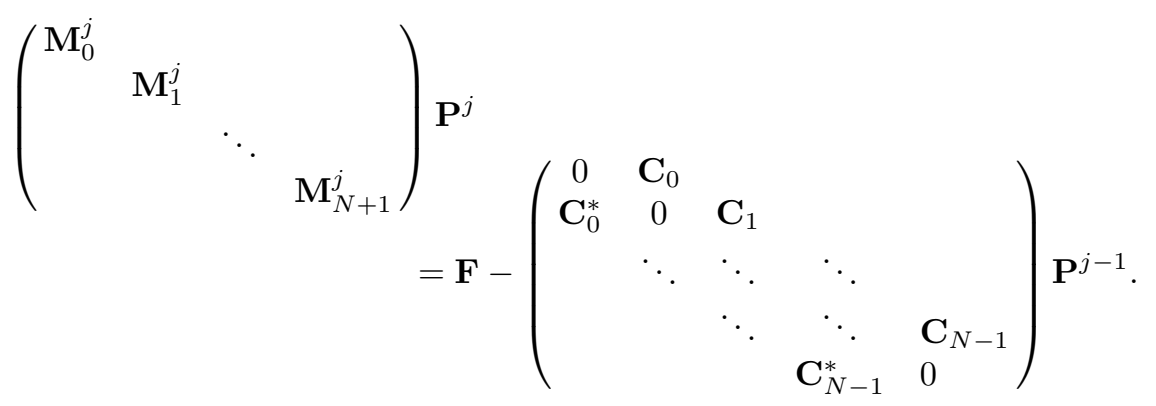

5. Convergence of the iterative schemes. This section provides a rudimentary convergence analysis of the iterative schemes described in section 4 .

THEOREM 5.1. There exists a constant $\epsilon>0$ such that if $\Delta t<\epsilon$, then the iterative methods (4.1), (4.2a), (4.2b), and (4.4) are all globally convergent. 
Proof. We prove the theorem for the concurrent update model scheme. Recall that the original, coupled system of equations take on the weak form:

$$
\begin{aligned}
\frac{1}{\Delta t}\left(\psi_{n}^{h}, v^{h}\right)+\left(i \Phi_{0 n}^{a} \psi_{n}^{h}, v^{h}\right)+\left(\left(2 \rho-1+\left|\psi_{n}^{h}\right|^{2}\right) \psi_{n}^{h}, v^{h}\right) \\
+\left(\left(i \operatorname{grad}+\mathbf{A}_{0 n}^{a}\right) \psi_{n}^{h},\left(i \operatorname{grad}+\mathbf{A}_{0 n}^{a}\right) v^{h}\right)-\rho\left(\psi_{(n+1)}^{h} \exp \left(-i \phi_{0 n}^{n+1}\right), v^{h}\right) \\
-\rho\left(\psi_{(n-1)}^{h} \exp \left(i \phi_{0(n-1)}^{n}\right), v^{h}\right)=\frac{1}{\Delta t}\left(\psi_{n, \text { old }}^{h}, v^{h}\right) \\
\forall v^{h} \in S^{h} \text { and for } n=0,1, \ldots, N .
\end{aligned}
$$

The concurrent update model (4.4), in its weak form, is given as

$$
\begin{gathered}
\frac{1}{\Delta t}\left(\psi_{n}^{j}, v^{h}\right)+\left(i \Phi_{0 n}^{a} \psi_{n}^{j}, v^{h}\right)+\left(\left(2 \rho-1+\left|\psi_{n}^{j}\right|^{2}\right) \psi_{n}^{j}, v^{h}\right) \\
+\left(\left(\text { igrad }+\mathbf{A}_{0 n}^{a}\right) \psi_{n}^{j},\left(i \operatorname{grad}+\mathbf{A}_{0 n}^{a}\right) v^{h}\right)-\rho\left(\psi_{(n+1)}^{j-1} \exp \left(-i \phi_{0 n}^{n+1}\right), v^{h}\right) \\
-\rho\left(\psi_{(n-1)}^{j-1} \exp \left(i \phi_{0(n-1)}^{n}\right), v^{h}\right)=\frac{1}{\Delta t}\left(\psi_{n, o l d}^{h}, v^{h}\right) \\
\forall v^{h} \in S^{h} \text { and for } n=0,1, \ldots, N .
\end{gathered}
$$

Taking the difference of these two expressions yields

$$
\begin{gathered}
\frac{1}{\Delta t}\left(\psi_{n}^{h}-\psi_{n}^{j}, v^{h}\right)+\left(i \Phi_{0 n}^{a}\left(\psi_{n}^{h}-\psi_{n}^{j}\right), v^{h}\right)+(2 \rho-1)\left(\psi_{n}^{h}-\psi_{n}^{j}, v^{h}\right) \\
+\left(\left|\psi_{n}^{h}\right|^{2} \psi_{n}^{h}-\left|\psi_{n}^{j}\right|^{2} \psi_{n}^{j}, v^{h}\right)+\left(\left(i \mathbf{g r a d}+\mathbf{A}_{0 n}^{a}\right)\left(\psi_{n}^{h}-\psi_{n}^{j}\right),\left(i \mathbf{g r a d}+\mathbf{A}_{0 n}^{a}\right) v^{h}\right) \\
=\rho\left(\left(\psi_{n+1}^{h}-\psi_{n+1}^{j-1}\right) \exp \left(-i \phi_{0 n}^{n+1}\right), v^{h}\right)+\rho\left(\left(\psi_{n-1}^{h}-\psi_{n-1}^{j-1}\right) \exp \left(i \phi_{0(n-1)}^{n}\right), v^{h}\right) \\
\forall v^{h} \in S^{h} \text { and for } n=0,1, \ldots, N .
\end{gathered}
$$

Letting $v^{h}=\psi_{n}^{h}-\psi_{n}^{j}=e_{n}^{j}$ in the above equation and considering only the real part, we get

$$
\begin{aligned}
\frac{1}{\Delta t}\left\|e_{n}^{j}\right\|_{L^{2}}^{2}+(2 \rho-1)\left\|e_{n}^{j}\right\|_{L^{2}}^{2}+\left\|\left(\operatorname{grad}+\mathbf{A}_{0 n}^{a}\right) e_{n}^{j}\right\|_{L^{2}}^{2} & \\
=\Re\left\{\rho\left(e_{n+1}^{j-1} \exp \left(-i \phi_{0 n}^{n+1}\right), e_{n}^{j}\right)+\rho\left(e_{n-1}^{j-1} \exp \left(i \phi_{0 n}^{n-1}\right), e_{n}^{j}\right)\right\} & -\left(\left|\psi_{n}^{h}\right|^{2} \psi_{n}^{h}-\left|\psi_{n}^{j}\right|^{2} \psi_{n}^{j}, \psi_{n}^{h}-\psi_{n}^{j}\right) .
\end{aligned}
$$

Taking advantage of the monotonicity found in the nonlinear term, one finds

$$
\left(\left|\psi_{n}^{h}\right|^{2} \psi_{n}^{h}-\left|\psi_{n}^{j}\right|^{2} \psi_{n}^{j}, \psi_{n}^{h}-\psi_{n}^{j}\right) \geq 0 .
$$

Then, it follows from Hölder's inequality that

$$
\begin{aligned}
\frac{1}{\Delta t}\left\|e_{n}^{j}\right\|_{L^{2}}^{2}+(2 \rho-1)\left\|e_{n}^{j}\right\|_{L^{2}}^{2} & +\left\|\left(\operatorname{igrad}+\mathbf{A}_{0 n}^{a}\right) e_{n}^{j}\right\|_{L^{2}}^{2} \\
& \leq \rho\left\|e_{n+1}^{j-1}\right\|_{L^{2}}\left\|e_{n}^{j}\right\|_{L^{2}}+\rho\left\|e_{n-1}^{j-1}\right\|_{L^{2}}\left\|e_{n}^{j}\right\|_{L^{2}} .
\end{aligned}
$$

This results in the expression

$$
\left((2 \rho-1)+\frac{1}{\Delta t}\right)\left\|e_{n}^{j}\right\|_{L^{2}}^{2} \leq \rho\left(\left\|e_{n+1}^{j-1}\right\|_{L^{2}}\left\|e_{n}^{j}\right\|_{L^{2}}+\left\|e_{n-1}^{j-1}\right\|_{L^{2}}\left\|e_{n}^{j}\right\|_{L^{2}}\right) .
$$


Summing over $n$ and applying Cauchy-Schwartz yields

$$
\left((2 \rho-1)+\frac{1}{\Delta t}\right) \sum_{n=0}^{N}\left\|e_{n}^{j}\right\|_{L^{2}}^{2} \leq \rho \sum_{n=0}^{N}\left(\left\|e_{n+1}^{j-1}\right\|_{L^{2}}\left\|e_{n}^{j}\right\|_{L^{2}}\right)+\rho \sum_{n=0}^{N}\left(\left\|e_{n-1}^{j-1}\right\|_{L^{2}}\left\|e_{n}^{j}\right\|_{L^{2}}\right)
$$

or

$$
\left((2 \rho-1)+\frac{1}{\Delta t}\right) \sum_{n=0}^{N}\left\|e_{n}^{j}\right\|_{L^{2}}^{2} \leq \rho\left(\sum_{n=0}^{N}\left\|e_{n}^{j-1}\right\|_{L^{2}}^{2}+\sum_{n=0}^{N}\left\|e_{n}^{j}\right\|_{L^{2}}^{2}\right) .
$$

Collecting like terms, one finds

$$
\left((\rho-1)+\frac{1}{\Delta t}\right) \sum_{n=0}^{N}\left\|e_{n}^{j}\right\|_{L^{2}}^{2} \leq \rho\left(\sum_{n=0}^{N}\left\|e_{n}^{j-1}\right\|_{L^{2}}^{2}\right) .
$$

From this, one obtains the desired error estimate:

$$
\sum_{n=0}^{N}\left\|e_{n}^{j}\right\|_{L^{2}}^{2} \leq \frac{\rho \Delta t}{\rho \Delta t+1-\Delta t} \sum_{n=0}^{N}\left\|e_{n}^{j-1}\right\|_{L^{2}}^{2}
$$

Thus, for $\Delta t<1$, the above multiplier is less than 1 and we have (linear) convergence for the concurrent update model. This convergence analysis can be generalized to encompass the other iterative schemes which were presented in section 4 as well. The major differences will be found in the mix of $e_{n}^{j}, e_{n-1}^{j-1}$, and $e_{n+1}^{j-1}$ terms of (5.3)-(5.5), but similar results are obtained.

Remark. Many factors are not considered in the above analysis such as the dependence of $\rho$ on the perpendicular coherence length $\xi_{\perp}$, the interlayer spacing, and the total number of layers $N$. More refined convergence analysis will be studied elsewhere.

Performance and efficiency benchmarks comparing the schemes in this section are given in section 6 .

6. Algorithmic comparisons. This section illustrates convergence properties observed for the respective algorithms for various simulations given a standard implementation model. Specifically, the numerical solution of the respective algorithms is computed by a finite element implementation using biquadratic basis functions over rectangular elements. The nonlinearity is solved using Newton's method. The resulting linear system, which is sparse and banded, is solved using a conjugate gradient method.

The physical sample of the model consists of six layers, each assumed to be homogeneous and isotropic and to have $\{x, y\}$-dimension $25 \xi_{\|} \times 25 \xi_{\|}$. The spacing (normal layer thickness) between the superconducting films is also taken to be $\xi_{\|}$. The material is assumed to be in a pure superconducting state when the sample is exposed to the external field defined by

$$
\vec{H}_{\text {ext }}=(0.07,0.07,0.125) \kappa
$$

and is allowed to settle into a steady-state vortex formation. The steady-state system is then subjected to a variable external field:

$$
\vec{H}_{\mathrm{var}}=\left(0.07,0.07,0.125-0.2\left(\frac{x-l / 2}{l}\right)\right) \kappa,
$$


TABLE 1

Benchmarks obtained for a fixed time step of $d t=0.2$.

\begin{tabular}{|c|c|c|c|c|}
\hline$d t=0.2$ & NPSM & PSM & RBM & CM \\
\hline \# Time steps & 26 & 26 & 75 & 107 \\
\hline \# Layer iterations & 156 & 157 & 451 & 964 \\
\hline \% Performance & Baseline & $100 \%$ & $288 \%$ & $412 \%$ \\
\hline
\end{tabular}

TABLE 2

Benchmarks obtained for a fixed time step of $d t=0.1$.

\begin{tabular}{|c|c|c|c|c|}
\hline$d t=0.1$ & NPSM & PSM & RBM & CM \\
\hline \# Time steps & 36 & 36 & 113 & 148 \\
\hline \# Layer iterations & 181 & 184 & 569 & 1039 \\
\hline \% Performance & Baseline & $100 \%$ & $314 \%$ & $411 \%$ \\
\hline
\end{tabular}

where $l$ is the length of the sample. This variable field induces a current within the sample. In the numerical implementation, each layer was decomposed into a $21 \times 21$ uniform rectangular grid.

The initial computations were performed on an isolated cluster of six PVMconnected DEC Alpha workstations located at the Advanced Computing Lab at Michigan State University. Benchmarks and timings of the algorithmic models of the previous sections are taken under the setting where the induced current causes motion and interaction of the vortices. Loosely speaking, the resulting implementation is well suited for a PVM implementation $[1,9,10]$ in that the problem exhibits large granularity. That is, the computational time required to solve the individual linear systems significantly outweighs the time required for exchanging solutions with neighboring layers when workstations are harnessed for the solution of the linear system. The tables below yield some indication of the speed of the respective algorithms. The data in Tables 1-3 were obtained by letting the computer models run over a fixed block of time in order to examine how "far" each algorithm was able to go. The first row of each table shows the number of time steps completed by the algorithm within the fixed block of time. The second entry in each table shows the total number of layer iterations required of the algorithm within the same block of time. The last entry in each table shows the percentage of time steps that the parallelized algorithm was able to calculate compared to the nonparallelized sequential method (NPSM).

In short, one sees that the parallelized sequential model (PSM) performs just slightly better than the NPSM. This is perhaps due to the fact that the initializations of the PSM are performed in parallel. These tasks include initialization of the order parameter, the generation of the vector magnetic potential, and the characterization of the geometry. Otherwise, the methods are identical. This does, however, show that the overhead introduced by the PVM implementation is somewhat insignificant in light of the small gains enjoyed by parallelizing the initialization routines.

Note also that the red-black method (RBM) was able to realize more than a threefold gain over the sequential method(s); this is contrary to what one might initially expect. On the other hand, the concurrent method (CM) showed a more moderate gain of slightly over four-fold.

Further examination of the above data leads to an indication of the computational efficiency of the respective algorithms. Examination of the number of layer iterations computed for each method shows that the number of layer iterations required per time step varies for each algorithm and for each fixed time step. Looking at the 
TABLE 3

Benchmarks obtained for a fixed time step of $d t=0.025$.

\begin{tabular}{|c|c|c|c|c|}
\hline$d t=0.025$ & NPSM & PSM & RBM & CM \\
\hline \# Time steps & 54 & 55 & 168 & 263 \\
\hline \# Layer iterations & 216 & 220 & 504 & 1052 \\
\hline \% Performance & Baseline & $102 \%$ & $311 \%$ & $487 \%$ \\
\hline
\end{tabular}

TABLE 4

A comparison of the number of iterations required to meet tolerance based on the step size dt.

\begin{tabular}{|c|c|c|c|c|c|c|c|c|}
\hline$d t$ & 0.025 & 0.05 & 0.1 & 0.2 & 0.3 & 0.4 & 0.5 & 0.75 \\
\hline Sequential & 4 & 4 & 5 & 6 & 7 & 8.5 & 10 & 12 \\
\hline Red-black & 3 & 4 & 5 & 6 & 6 & 8 & 9 & 12 \\
\hline Concurrent & 4 & 5 & 7 & 9 & 10 & 14 & 16 & 21 \\
\hline
\end{tabular}

number of layer iterations required for each time step, one sees that the CM generally required more layer iterations to reach tolerance at a given time step whereas the RBM generally required the least. A comparison of the required number of layer iterations for convergence is given in Table 4 . Table 4 shows the number of layer iterations required to meet tolerance per time step as a function of the step size taken in time for each of the respective algorithms. In other words, Table 4 shows the number of iterations required to reach convergence in (4.1), (4.2a), (4.2b), and (4.4), respectively, based on the value of $\Delta t$ in (3.3). Note that the two sequential methods, the NPSM and PSM, yield equivalent solutions; therefore, they need not be considered independently. From the table, one sees that the RBM required the least number of layer iterations to converge to the specified tolerance while the $\mathrm{CM}$ required the most layer iterations to converge.

Similar numerical tests were conducted on a 128-node Intel Paragon located at the Hong Kong University of Science and Technology. The data in Table 5 were obtained by running PSM, RBM, and CM for the vortex torsion problem described in section 7. The total number of layers in the simulation is 10 and the maximum number of nodes used is 16 . When the number of layers was increased to 40 , while the interlayer spacing was reduced by a factor of 0.25 , the corresponding results are given in Table 6 and Table 7. For the latter two simulations, a maximum of 46 processors was used for the concurrent computation.
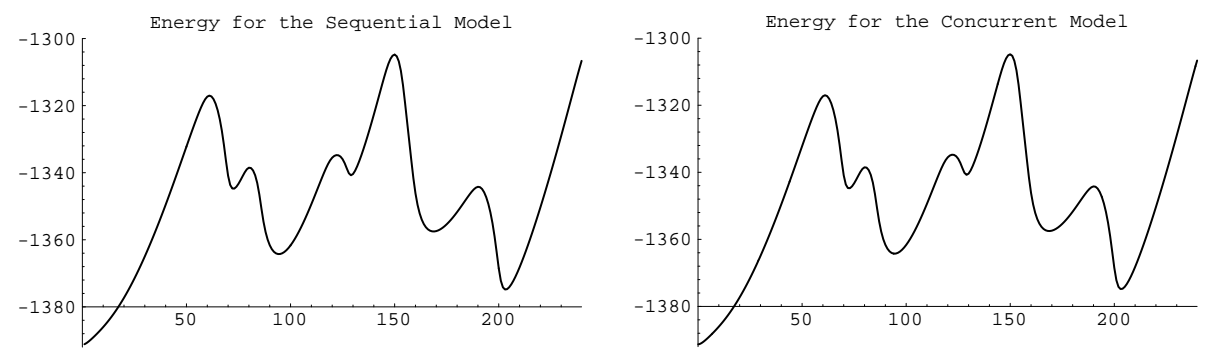

FIG. 3. Energy vs. time for the sequential and concurrent schemes. The close resemblance substantiates that the solutions of the two algorithms yield consistent information. 
TABLE 5

Benchmarks obtained on the Intel Paragon for a sample with 10 layers and dt=0.2.

\begin{tabular}{|c|c|c|c|}
\hline$d t=0.2$ & PSM & RBM & CM \\
\hline \# Time steps & 15 & 90 & 151 \\
\hline \# Layer iterations & 60 & 271 & 528 \\
\hline \% Performance & Baseline $\%$ & $600 \%$ & $1007 \%$ \\
\hline
\end{tabular}

TABLE 6

Benchmarks obtained on the Intel Paragon for a sample with 40 layers and $d t=0.2$.

\begin{tabular}{|c|c|c|c|}
\hline$d t=0.2$ & PSM & RBM & CM \\
\hline \# Time steps & 1.5 & 35.5 & 66 \\
\hline \# Layer iterations & 10 & 214 & 462 \\
\hline \% Performance & Baseline $\%$ & $2367 \%$ & $4400 \%$ \\
\hline
\end{tabular}

Computational comparisons aside, there is still a question of consistency between the methods. A succinct indication of how the results of the respective methods compare with each other is seen in the energy of the respective systems. Figure 3 shows the energies associated with the computational solutions of the extreme cases: the simultaneous algorithm and the concurrent algorithm. The close resemblance indicates that the two algorithms are indeed consistent in their calculation of the solution of the model. The consistency of the algorithms is further substantiated by Figure 4. Figure 4 shows a snapshot of the vortex state after 1200 steps in time as calculated by the sequential method (SM) and the CM. The shadow plots of the vortex tubes are created by Mathematica from the data set representing the magnitude of the order parameters in all the layers. The vortex location corresponds to where the order parameter vanishes. The vortex states of the two systems are nearly indistinguishable.

7. Physical interpretations. Our objective is the accurate depiction of vortex dynamics which can aid in material design or provide better insight into and characterization of vortex dynamics. This section serves as a brief illustration as to how we use the algorithms discussed in the previous sections for various physical simulations. The physical simulations presented here include the visualizations of vortex dynamics as they are influenced by normal inclusions and induced currents.

7.1. Variable fields/induced currents. The numerical benchmarks presented in section 6 were obtained for the setting where the sample -initially in a pure superconducting state - was subjected to an applied field and allowed to reach a new steady-state configuration. This steady-state configuration was then subjected to the variable applied field (6.2). The variable field induced a current within the sample, driving the vortices across the sample. The energy plots shown in Figure 3 show various peaks and dips in the energy associated with the system. These variations in the energy of the system are related to vortices being created and vortices exiting the region. The induced current causes the vortices to move (somewhat uniformly) across the sample. This allows room for the generation of new vortices and causes vortices to be pushed out of the superconducting region. Figure 5 provides a series of snapshots which illustrate the phenomena just described of a vortex being generated. The three-dimensional plots were created by Mathematica from the data set representing the magnitude of the order parameters in all layers.

7.2. Stub pinning walls. The computational results given in this next simulation were derived from modeling a material consisting of 12 layers. The supercon- 
TABle 7

Benchmarks obtained on the Intel Paragon for a sample with 40 layers and $d t=0.02$.

\begin{tabular}{|c|c|c|c|}
\hline$d t=0.02$ & PSM & RBM & CM \\
\hline \# Time steps & 3 & 73.3 & 135.5 \\
\hline \# Layer iterations & 12 & 220 & 472 \\
\hline \% Performance & Baseline \% & $2443 \%$ & $4517 \%$ \\
\hline
\end{tabular}
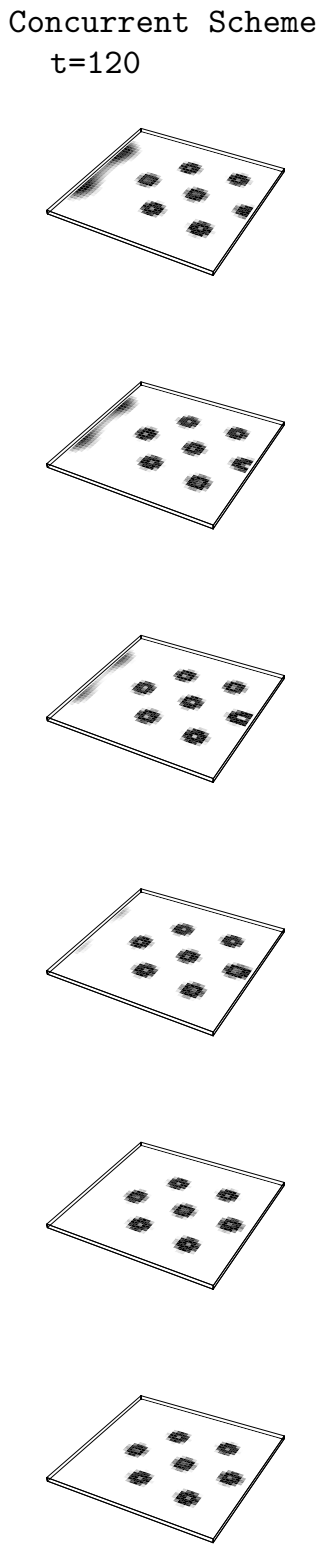

$$
\begin{gathered}
\text { Sequential Scheme } \\
t=120
\end{gathered}
$$
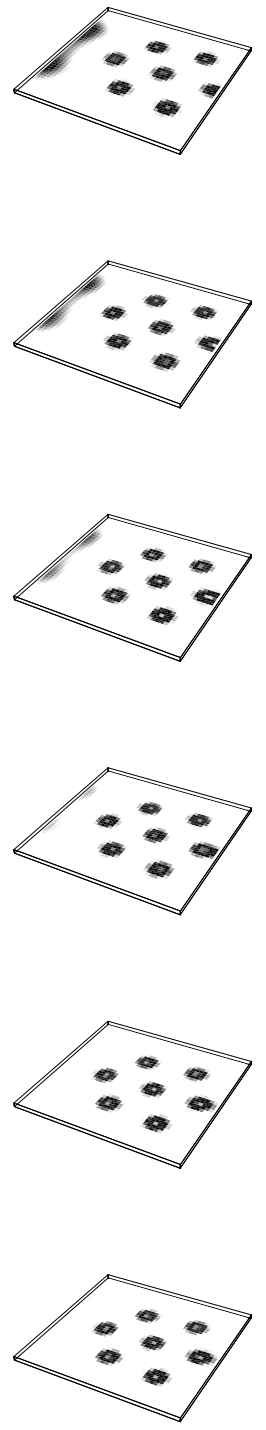

FIG. 4. Snapshots of the vortex states as calculated by the sequential method (left) and the concurrent method (right). Their close resemblance supports the consistency of the two algorithms. 

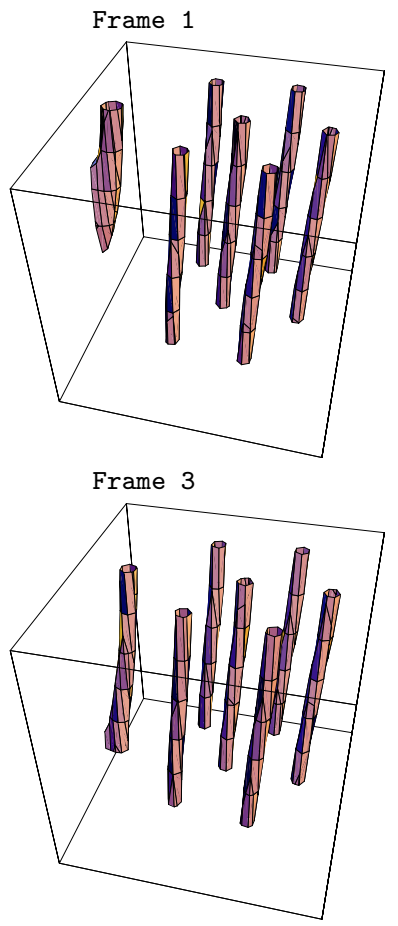

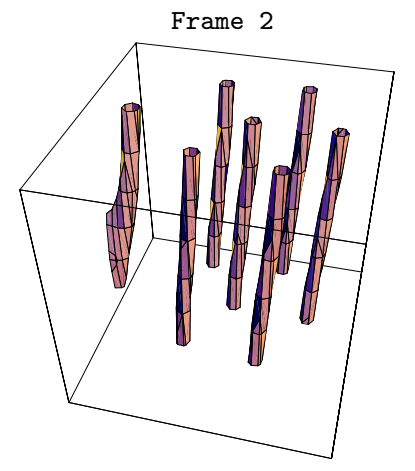

Frame 4

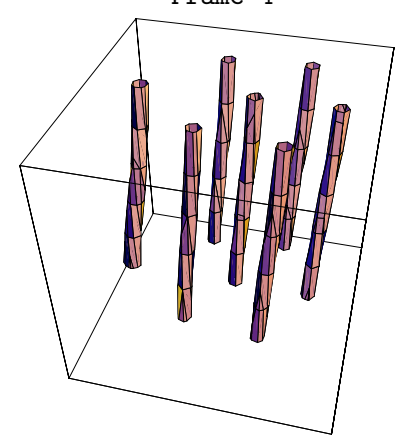

FIG. 5. Snapshots of the creation of the vortex states.
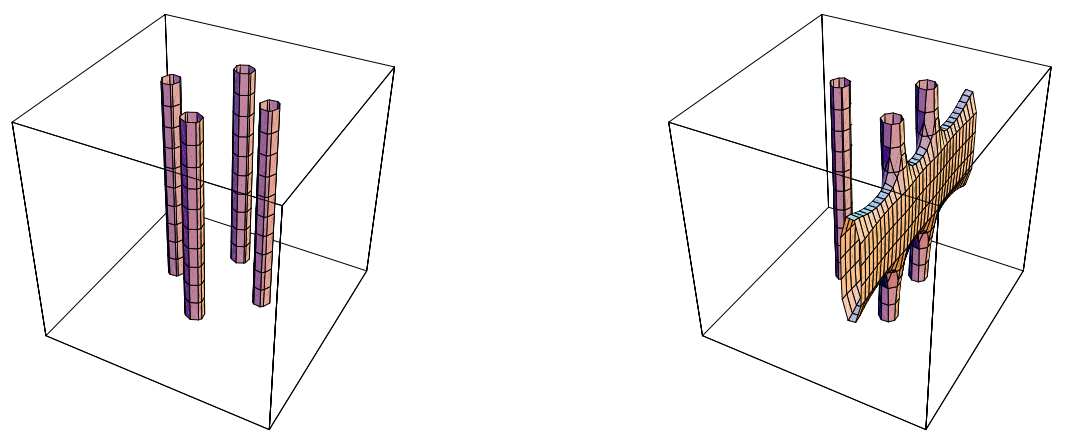

FIG. 6. Snapshots of the steady-state configuration of the vortex states.

ducting layers are square, measuring $25 \xi_{\|} \times 25 \xi_{\|}$, the spacing between the layers is taken to be $\xi_{\|}$, and the initial state of the system is taken to be the steady state corresponding to the external applied field

$$
\vec{H}_{\text {ext }}=(0,0,0.05) \kappa .
$$

When the material reaches steady state in the absence of inhomogeneities in the material, the vortex tubes become stationary, as shown in the left figure of Figure 6 .

In the presence of a pinning wall, the placement of the vortex tubes becomes that of the right figure in Figure 6, where there are three vortices present in the steady state, two of which have become entwined with the pinning wall. The vortex wall consists of normal material placed within the sample from layers 4 through 8 , with a width of $1 \frac{1}{2}$ coherence lengths and 10 coherence lengths from the boundary. 

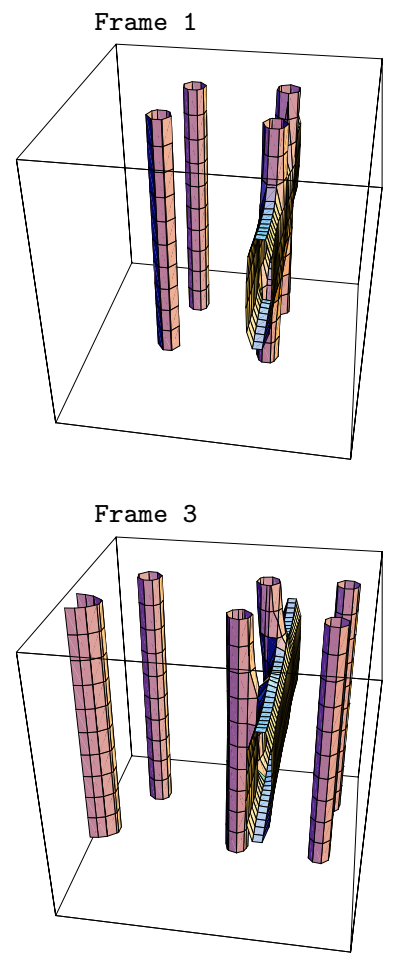
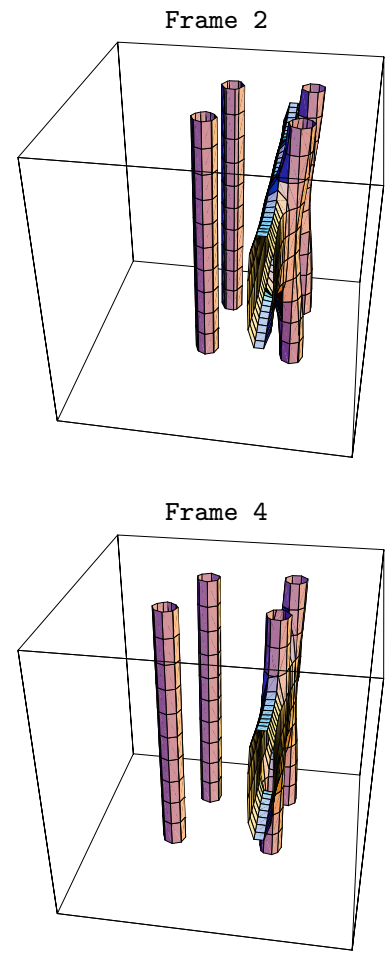

FIG. 7. Motion of the vortices induced by the applied current.

Behavior of this system under an induced current is also of interest. If the system is subjected to a varying applied field, a current is introduced into the system which causes the vortices to move across the sample. The next result shows what happens to the above steady-state system (with a pinning wall) when the sample is subjected to the varying field:

$$
\vec{H}_{\mathrm{var}}=\left(0,0,0.05-0.1\left(\frac{x-l / 2}{l}\right)\right) \kappa,
$$

where $l$ is the length of the sample.

The induced motion of the vortices causes the vortices attached to the pinning wall to become dislodged. Figure 7 shows characteristic behavior and interactions between the vortices and with the stub pinning wall.

7.3. Vortex torsion. The numerical benchmarks on the Intel Paragon were obtained for the setting where the sample was initially in a superconducting state and was subjected to an external magnetic field perpendicular to the layers. The $\{x, y\}$-dimension of each layer is $10 \xi_{\|} \times 10 \xi_{\|}$and the interlayer spacing is $2 \xi_{\|}$for the case with a total of 10 layers and $0.5 \xi_{\|}$for the case with a total of 40 layers. The external field is defined by

$$
\vec{H}_{\text {ext }}=(0,0,0.35) \kappa .
$$

A periodic boundary condition in the $z$-direction is used. The normal inclusions are introduced to the LD models in ways similar to that in [3] and [16], i.e., modifying 


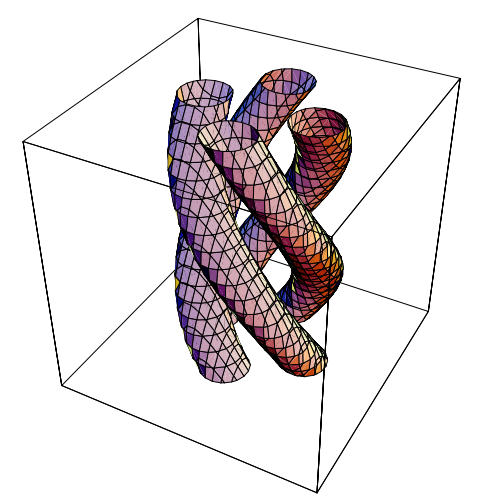

FIG. 8. Vortex torsion due to normal inclusions.

the term

$$
-\psi_{0 n}+\left|\psi_{0 n}\right|^{2} \psi_{0 n}
$$

in (3.1) by

$$
-\alpha_{n}(x, y) \psi_{0 n}+\left|\psi_{0 n}\right|^{2} \psi_{0 n}
$$

The variation of $\alpha_{n}(x, y)$ can be used to simulate the effect of thermal fluctuation or the modulation of $T_{c}$. If $\alpha_{n}(x, y)$ is negative in certain regions, we refer to it as a normal inclusion. When the normal inclusions are absent, the vortex lines are straight lines parallel to the $z$-axis. In Figure 8 , torsion of the vortices was obtained. The vortices in the picture are periodic extensions of the numerical solution for the 10 layers. The cores of the vortex lines are located precisely where normal inclusions are placed. Other simulations involving tilted applied fields, current, and pinning may be found in [12] and further investigations involving combinations of tilted applied fields, splayed pinning sites, and applied fields are also warranted. Further numerical experiments will be carried out to complement the theoretical studies in [5] and other literatures on the self-induced motion as well as the motion due to an applied current of three-dimensional vortex lines in anisotropic samples.

8. Conclusion. The LD models have been used to study the three-dimensional vortex dynamics as well as the three-dimensional-two-dimensional crossover in layered superconductors. A number of parallel algorithms discussed here take advantage of the existing GL codes and modern distributed architectures and facilitating software. They overcome the computational complexity and make simulations of physical phenomena possible. Simple tests have been presented here and more extensive experiments will be conducted in collaborations with physicists in the future. Other parallelization strategies like the coupling of the nonlinear iteration with the layer updates and domain decomposition techniques will also be explored. Parallel implementation in other environments such as the message passing interface setting are also under development.

Acknowledgments. The authors wish to thank Dr. J. Clem of the Ames Laboratory and Iowa State University and Dr. R. Klemm of the Argonne National Laboratory for helpful discussions of the physical problems. The authors also wish to thank 
Dr. L. Ni and the Advanced Computing Lab at Michigan State University for the use of their facility.

\section{REFERENCES}

[1] A. Beguelin, J. Dongarra, A. Geist, W. Jiang, R. Manchek, and V. Sunderam, PVM: Parallel Virtual Machine, A User's Guide and Tutorial for Networked Parallel Computing, MIT Press, Cambridge, MA, 1994.

[2] L. BulaevskiI, Magnetic properties of layered superconductors with weak interaction between the layers, Zh. Eksperim. i Teor. Fiz., 64 (1973), pp. 2241-2247 (in Russian); Soviet Phys.-JETP, 37 (1973), pp. 1133-1136 (in English).

[3] S. J. Chapman, Q. Du, and M. D. Gunzburger, A Ginzburg-Landau type model of superconducting/normal junctions including Josephson junctions, European J. Appl. Math., 6 (1995), pp. 97-114.

[4] S. J. Chapman, Q. Du, and M. D. Gunzburger, On the Lawrence-Doniach and anisotropic Ginzburg-Landau models for layered superconductors, SIAM J. Appl. Math., 55 (1995), pp. $156-174$.

[5] S. J. Chapman And G. Richardson, Motion and homogenization of vortices in anisotropic type-II superconductors, SIAM J. Appl. Math., 58 (1998), pp. 587-606.

[6] Q. Du And P. Gray, High-kappa limits of the time-dependent Ginzburg-Landau model, SIAM J. Appl. Math., 56 (1996), pp. 1060-1093.

[7] Q. Du, M. D. Gunzburger, and J. S. Peterson, Analysis and approximation of the Ginzburg-Landau model of superconductivity, SIAM Rev., 34 (1992), pp. 54-81.

[8] Q. Du, M. D. Gunzburger, And J. S. Peterson, Computational simulation of type-II superconductivity including pinning phenomena, Phys. Rev. B, 1995.

[9] J. Garner, M. Jones, and P. Plassmann, Parallel algorithms for modeling superconductors, in Computing at the Leading Edge, A. Mirin, ed., publication UCRL-TB-111084, National Energy Research Supercomputer Center, Lawrence Livermore National Laboratory, Livermore, CA, 1993.

[10] G. A. Geist and V. S. Sunderam, The PVM system: Supercomputer level concurrent computation on a heterogeneous network of workstations, in Proceedings of the Sixth Distributed Memory Computing Conference, IEEE, 1991, pp. 258-261.

[11] P. Gray, Superconductivity Models and their Numerical Implementation, Ph.D. thesis, Michigan State University, E. Lansing, MI, 1996.

[12] P. GRAY, Iteration schemes for parallelizing models of superconductivity, in Proceedings of the 1996 Copper Mountain Conference on Iterative Methods, University of Colorado, Boulder, CO, 1996.

[13] Y. IYE, How anisotropic are the cuprate high Tc superconductors?, Comments Cond. Mat. Phys., 16 (1992), pp. 89-111.

[14] R. Klemm, A. Luther, And M. Beasley, Theory of the upper critical field in layered superconductors, Phys. Rev. B, 12 (1975), pp. 877-891.

[15] W. LaWrence and S. Doniach, Theory of layer structure superconductors, in Proceedings of the 12th International Conference on Low Temperature Physics, Academic Press of Japan, Kyoto, 1971, pp. 361-362.

[16] M. MAChida AND H. KABURAKI, Direct numerical experiment on two-dimensional pinning dynamics of a three-dimensional vortex line in layered superconductors, Phys. Rev. Lett., 75 (1995), pp. 3178-3181.

[17] M. Tinkham, Introduction to Superconductivity, 2nd ed., McGraw-Hill, New York, 1994

[18] P. YANG AND C. Lieber, Nanorod-superconductor composited: A pathway to high critical current density materials, Science, 273 (1996), p. 1836. 